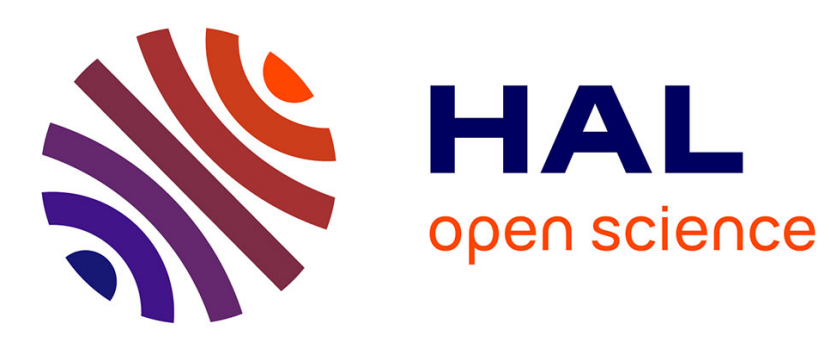

\title{
Stability of a directional Marangoni flow.
}

Corentin Tregouët, Arnaud Saint-Jalmes

\section{To cite this version:}

Corentin Tregouët, Arnaud Saint-Jalmes. Stability of a directional Marangoni flow.. Soft Matter, 2020, 16 (38), pp.8933-8939. 10.1039/D0SM01347A . hal-02929048

\section{HAL Id: hal-02929048 \\ https://hal.science/hal-02929048}

Submitted on 3 Sep 2020

HAL is a multi-disciplinary open access archive for the deposit and dissemination of scientific research documents, whether they are published or not. The documents may come from teaching and research institutions in France or abroad, or from public or private research centers.
L'archive ouverte pluridisciplinaire HAL, est destinée au dépôt et à la diffusion de documents scientifiques de niveau recherche, publiés ou non, émanant des établissements d'enseignement et de recherche français ou étrangers, des laboratoires publics ou privés. 


\title{
Stability of a directional Marangoni flow.
}

\author{
Corentin Tregouet, ${ }^{* a}$ and Arnaud Saint-Jalmes ${ }^{a}$ \\ Marangoni flows result from surface-tension gradients, and \\ these flows occur over finite distances on the surface, but the \\ subsequent secondary flows can be observed on much larger \\ lengthscales. These flows play major roles in various pheno- \\ mena, from foam dynamics to microswimmer propulsion. We \\ show here that if a Marangoni flow of soluble surfactants is \\ confined laterally, the flow forms an inertial surface jet. A full \\ picture of the flows on the surface is exhibited, and the velocity \\ profile of the jet is predicted analytically, and is successfully \\ compared with the experimental measurements. Moreover, this \\ straight jet eventually destabilizes into meanders. A quanti- \\ tative comparison between the theory and our experimental \\ observations yields a very good agreement in terms of critical \\ wavelengths. The characterization and understanding of the \\ 2D flows generated by confined Marangoni spreading is a first \\ step to understand the role of inertial effects in the Marangoni \\ flows with and without confinement.
}

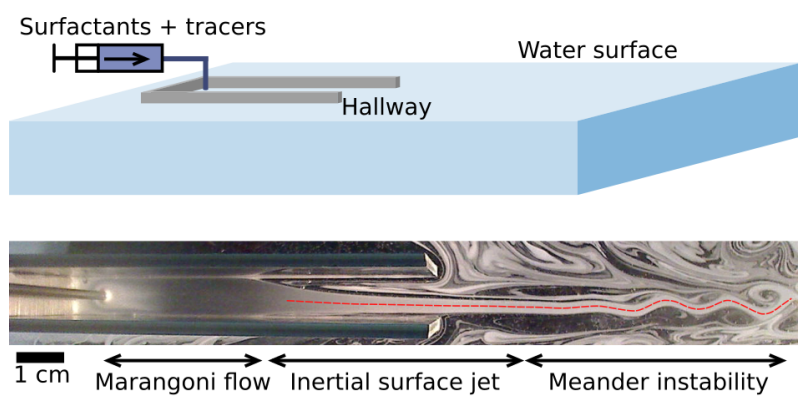

a Univ Rennes, CNRS, IPR (Institut de Physique de Rennes), UMR 6251, F-35000, Rennes, France. E-mail : corentin.tregouet@univ-rennes1.fr

\section{Introduction}

Marangoni flows can be ignited at a fluid interface by the occurrence of a surface-tension gradient : they typically spread over centimeters at the surface, while their vertical extent remains of the order of a millimeter. They are characterized by their heights which are negligible compared to their horizontal extents, and they can therefore be considered as a 2D- (or surface-) flows.

At the everyday life scale, Marangoni flows are actually easy to trigger and are often invoked to explain many apparently simple observations. Indeed, Marangoni effects are crucial in coating processes, or liquid-film extraction out of a bath in relation with foaming 1, 2, 3, 4. Similarly, they also play a major role in liquid-film rupture and antifoaming phenomena, 5, 6, 7, 8, 9, as well as in droplet spreading [10, 11, 12, and even in the propulsion of small objects such as swimming droplets [13, solid microswimmers [14, or even insects [15, 16].

Consequently, fundamental analyses of these surface flows have attracted a significant attention [17, 18, 19, 20], and found direct applications in material design 21] and microfluidics 22]. Besides, another important feature of $2 \mathrm{D}$ flows is that they spontaneously create complex patterns with swirls and coils, and even ending up to twodimensional turbulence. The occurrence of such turbulent flows has been reported in soap films [23, 24, 25, while other instabilities can be triggered by circular Marangoni flows [26].

Despite all the attention gathered by such phenomena in the community, a fundamental but yet unanswered questions still holds : what is the extent of a Marangoni flow created by a point source, and how it depends on surfac-

tant properties. In other words, a central question in the different applications remains, which is to understand how and how far a directional Marangoni flow extends, especially in relation with surface micro-swimmers.

Experiments [17, 26, 18, 27, 28 and simulations [19, 20. show that fast Marangoni flows only extend on a finite radius (for radial flows) or length (for directional flows), which can be understood thanks to a power-law analysis balancing in-plane advection and out-of-plane diffusion [26, 18]. Beyond this fast spreading, slower unstable secondary flows can be observed, which exhibit pluming instabilities [26, 18]. These secondary flows are also limited in space in a radius of the same order of magnitude as the primary Marangoni flow. These instabilities could be the key to understand the discrepancy between the experiments and the analytical theory that predicts that the Marangoni flow asymptotically tends to zero far away from the source 29. Fully understanding this instability would require more theoretical analysis of the flow stability [30], and more experiments.

In this paper, we bring first elements to understand the effect of this pluming phenomenon by studying the flow in the absence of the usual pluming instability. By confining the flow laterally, we obtain a slow directional surface flow which extends beyond the fast Marangoni spreading length. The other interest of this study is to analyze a flow mimicking the flow created behind asymmetrical Marangoni micro-swimmers. We first study a uniaxial (directional) Marangoni flow in which we prevent the pluming instability visible in [26, 18, to develop by confining laterally the flow on a width smaller than the plume width. The method to obtain such a flow is detailed in Section 2. We show in Section 3 that the obtained surface flow extends on a very long distance compared to what is observed without lateral 
confinement. Moreover, a meandering instability appears along the flow. We explain in Section 4 that the inertia of the boundary layer and its slow diffusive growth are responsible for the extension of the Marangoni flow into an inertial jet, and that the observed meandering instability is due to a specific kind of Kelvin-Helmholtz instability described by Rayleigh.

\section{Material and methods}

\section{Condition and set up for the flow generation}

A soluble surfactant solution is deposited on an air/water interface confined on three sides by a long hallway closed in one end, as shown in Figure 1

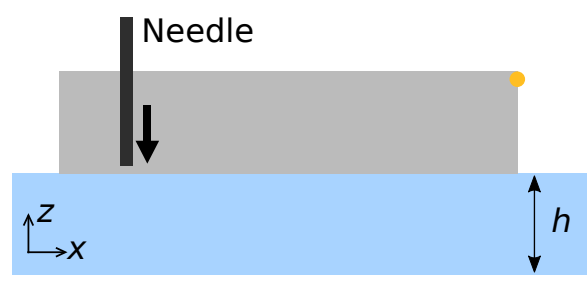

(a) Side view: horizontal projection

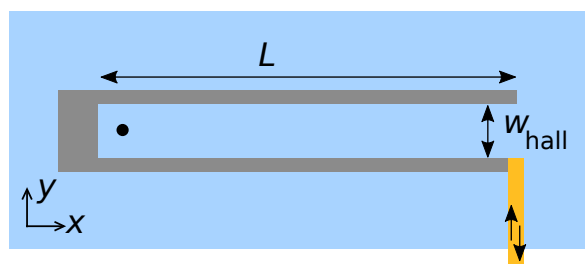

(b) Top view: vertical projection

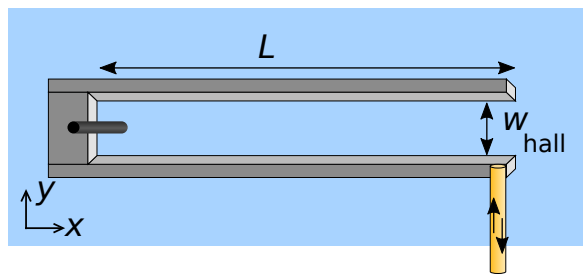

(c) Top view: perspective projection

Figure 1 - Setup used to generate the surface jet. Thin walls (gray) are hanged over a water bath (blue), and a metal needle (black) is used to spread the surfactant solution on the surface. One of the walls is excited with a beam (orange) connected to a loudspeaker. (a) : side view. (b) : top view. (c) : perspective view.

Fresh millipore water is used for the bath and to prepare the surfactant solution. A daily-made solution of Sodium Dodecyl Sulfate (SDS) concentrated at 3 times the critical micellar concentration (CMC) is deposited with a Harvard Apparatus syringe pump on the air/water interface at a flow rate of typically $Q=0.6 \mathrm{~mL} / \mathrm{min}$ (molar flow rate of $\left.q_{\mathrm{m}}=12.2 \mu \mathrm{mol} / \mathrm{min}\right)$. The hallway is formed around the point of deposition by three walls hanged over the bath to be just in contact with the water. The needle used as surfactant injector is placed just over the surface, at the closed end of the hallway. The length $L$ of the hallway is between $30 \mathrm{~mm}$ and $100 \mathrm{~mm}$.

A comprehensive study of the pluming instability visible without confinement in [26, 18, will be the topic a future work, but we observed that the wavelength is close to the depth of the bath. Accordingly, to hinder the development of this instability, we confine laterally the flow on a width $w_{\text {hall }}$ of $12 \mathrm{~mm}$ or $6 \mathrm{~mm}$, while the water height $h$ is always greater than $30 \mathrm{~mm}$. This also prevents any interaction between the possible vertical vertices 31 and the bottom. Some experiments were performed in shallow water ( $h<10 \mathrm{~mm}$ ) for comparison, and no noticeable difference in the flow morphology was observed.

The confinement is effective if the length of the fast Marangoni flow (the spreading length $L_{\mathrm{Mar}}$ ) respects some geometrical constraints : $w_{\text {hall }} / 2<L_{\text {Mar }}<L$. This raises strong conditions on the molar flow rate, which has a strong effect on the spreading length $L_{\text {Mar }} \propto q_{\mathrm{m}}^{3}[18$. The molar flow rate and hence the flow speed $\left(v \propto q_{\mathrm{m}}^{-1}\right)[18$ are therefore limited in a reduced range by the geometrical conditions.

Additionally, for the phenomenon to be well controlled, the surface and the boundary layers must be stable. This requires to maintain low capillary $\left(\mathrm{Ca}=\frac{\eta v}{\gamma}\right)$ and Reynolds numbers $\left(\mathcal{R} e=\frac{\rho v L}{\eta}\right)$, where $\eta, v, \rho, L$ and $\gamma$ are respectively the viscosity, the velocity, the density, the characteristic length, and the surface tension. But neither the velocity nor the length are controlled directly : both result from the diffusion of the surfactants from the surface to the bulk, and from the molar flowrate of surfactants.

\section{Velocity mapping on the surface}

Images of the surface are acquired with a USB camera Mako U-130B mounted with a $25 \mathrm{~mm}$ lens from Edmund Optics, enabling a resolution of $5.5 \mathrm{pixel} / \mathrm{mm}$. All the images are analyzed with ImageJ, and the particle tracking is made by the Python module trackpy.

A first type of tracer is used to identify the regions of high velocity : the surfactant solution is emulsified with sunflower oil to create an oil-in-water emulsion, with a 1to-1 oil/water ratio. The oil droplets (of diameter in the micrometer range) are used as surface tracers, as in some previous studies on Marangoni flows [26, 18]. Because of the oil droplets and the lighting from the top, the gray levels on the flow image are an indication of the velocity : when the flow is accelerated it tends to dilute the droplets, letting the dark background appear, while when the flow gets slower, the droplets concentrate and the image becomes white.

To reveal the streamlines on the surface, a second type of tracers is used : ground pepper is deposited on the surface, coupled with grazing-incidence lighting. Unlike the oil droplets, these tracers are large enough to be individually visible on the images, enabling particle tracking, and they are dispersed on the surface prior to jet creation. Image correlation enables to calculate the velocity on every point of the surface. However, the large variations of velocity between 


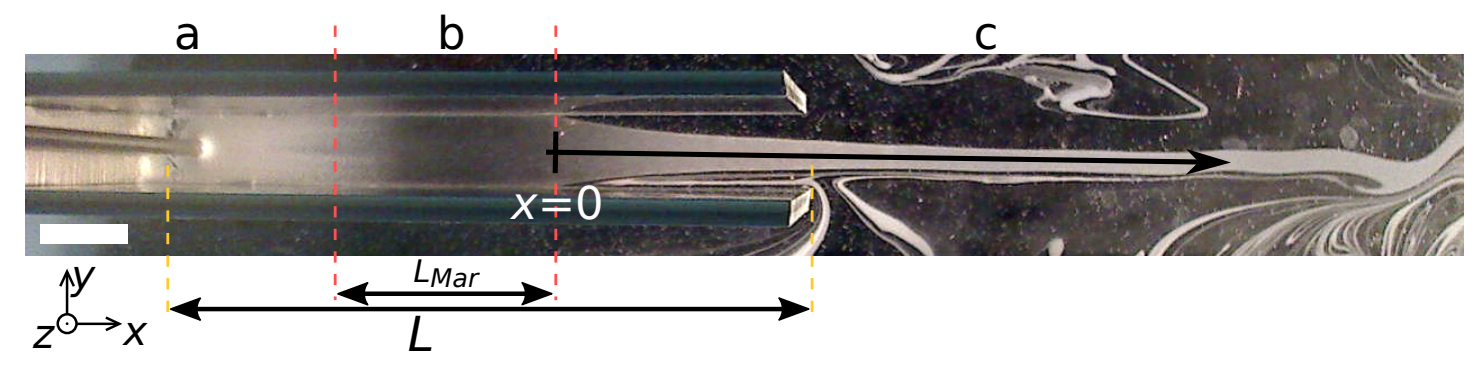

Figure 2 - The different steps of the uniaxial-flow generation (no excitation) (top view) : a- deposition of surfactant and emulsion ; b- acceleration due to the Marangoni effect on a length $L_{\mathrm{Mar}}$ (the acceleration leads to the dilution of the emulsion and darker colors); c- jet : deceleration and focusing; $q_{\mathrm{m}}=14.2 \mu \mathrm{mol} / \mathrm{min}$. Scale bar : $10 \mathrm{~mm}$.

the jet and the rest of the surface prevent this method to be used for the jet itself. The jet velocity can be measured to complete the previous measurement, by seeding individual ground-pepper particles directly in the flow, very carefully not to disturb or even stop the jet. Despite their hydrophobic character, ground-pepper flakes have been shown to be ideal non-inertial tracers 32, and there are used here with a packing fraction of typically $5 \%$.

In the first case, the morphology of the flow is clearly visible, and the instability (and its wavelength) appears at first sight, but only the second type of tracer solution allows for precise velocity measurements.

\section{Forced destabilization of the flow}

In order to analyze the uniaxial-flow stability regarding the meander instability, one of the lateral wall is fixed to a loudspeaker, which will be used in the second part of this work to create small perturbations on the flow at specific frequencies, as shown in Figure 1. The loudspeaker is powered with a sinusoidal wave at a fixed frequency, and a movie is recorded at the same frequency to facilitate the observation of the excited mode with droplet tracers.

Using stroboscopic imaging, the meanders seem to be at constant positions when they result from the amplification of the initial perturbation (unstable frequencies). On the contrary, if the initial perturbation is stable, its amplitude decreases exponentially. Other frequencies then develop spontaneously, but are not synchronized with the camera acquisition rate set to the forcing frequency, so they appear mobile on the movie. Under stroboscopic imaging, a fixed (moving) pattern is therefore the evidence that the forced frequency is unstable (stable).

\section{Morphology of the uniaxial flow}

The uniaxial-flow shows the same steps as what has been observed by Le Roux et al. 18, as illustrated in Figure 2 with the use of droplet tracers : $(a)$ - the source, where the surfactant is deposited, $(b)$ - a Marangoni finite spreading length $L_{\text {Mar }}$ which is typically around $50 \mathrm{~mm}$ and described and analyzed in 18, and $(c)$ - a sharp deceleration beyond. As $w_{\text {hall }}<h$, no pluming instability is observed after $L_{\mathrm{Mar}}$, and the moving fluid continues in a straight line even beyond the confined area. For this reason, we call this straight surface flow a surface jet.

\section{Velocity field on the surface}

Particle tracking is used to investigate the velocity field on the surface. The superimposition of treated images is presented in Figure $3 \mathrm{a}$ : it is clearly visible that the streamlines converge.

The corresponding velocity map is presented in Figure $3 \mathrm{~b}$. On this map, the jet is visible as the region where velocity is too high and the profile too steep to be measured with this method (between black lines). However, the velocity profile inside the jet has been measured separately in the same flow conditions as in Figure $3 \mathrm{a}, \mathrm{b}, \mathrm{c}$, and is presented in Figure $3 \mathrm{~d}$. It shows a very quick acceleration and a plateau, in agreement with the grey level in Figure 2, and then a slow deceleration, from $170 \mathrm{~mm} / \mathrm{s}$ to a few tens of $\mathrm{mm} / \mathrm{s}$.

Around the jet, we observe lateral zones which represent the linear growth of the region in motion (dashed lines are guides for the eyes). An analysis of the velocity profiles around the jet as shown in Figure 3. highlights the observation that lateral extension of the velocity profile increases along the trajectory.

Just at the exit of the hallway, the jet width meets a minimum, which corresponds to the actual initial jet width. This width is measured to be $w_{\text {tracking }}=6.8 \mathrm{~mm}$ in Figure $3 \mathrm{~b}$. After this minimum, the jet-width grows along the flow, as shown in Figure $3 \mathrm{~b}$.

\section{Meandering patterns}

In the region $c$ of Figure 2, the jet always destabilizes in meanders with a well-defined wavelength. The instability then amplifies until destruction of the jet and eddies can be observed in the outer sides of these meanders as reported in Figures $4 \mathrm{a}, 4 \mathrm{~b}, 4 \mathrm{c}$ and 5 a (droplet tracers).

This destabilization triggers the destruction of the jet which mixes with the whole surface creating the patterns accumulating on the surface of finite size, and observed around the jet in Figures $4 \mathrm{p}$ and 4 . 
(a)

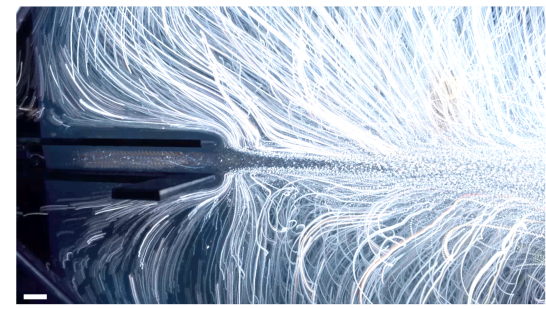

(b)

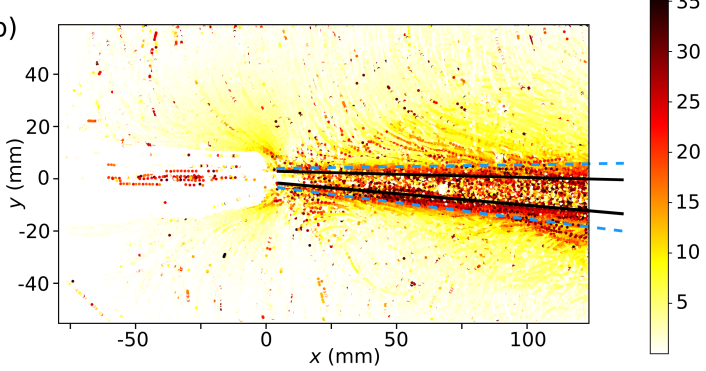

(c)

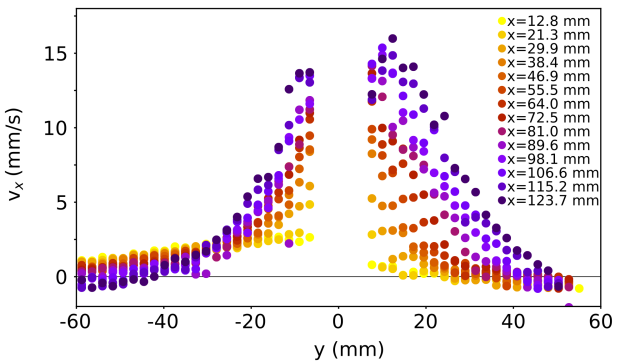

(d)

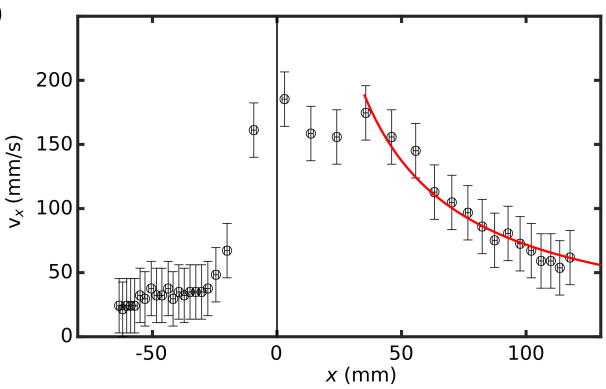

FigURE 3 - Particle tracking with $w_{\text {hall }}=12 \mathrm{~mm}$ and $q_{\mathrm{m}}=12.2 \mu \mathrm{mol} / \mathrm{min}$ : (a)- the superposition of images lets the streamlines appear. Scale bar : $10 \mathrm{~mm}$. (b)- the $x$-velocity map (in $\mathrm{cm} / \mathrm{s}$ ) shows the extension of the jet during convergence of the streamlines. Guides : black lines delimit the jet, dashed blue lines highlights the extension of the areas driven into motion by the jet. (c) Velocity profile along cutlines perpendicular to the flow, for different positions along the flow from upstream (yellow) to downstream (purple). (d) Velocity of a particle in the middle of the jet. Red line : best fit using Eq (9), with $v_{0}=189 \mathrm{~mm} / \mathrm{s}, \alpha^{-1 / 2} \delta_{0}=0.433 \mathrm{~mm}$ in $x_{0}=35 \mathrm{~mm}$.

Experiments without tracers observed by surfacedeformation detection showed that the jet and the instability exist without tracers.

The sinusoidal perturbation of the flow enables a stability analysis of individual modes in the range of interest using a loudspeaker to create the perturbations observed with stroboscopic imaging. High frequencies (short wavelengths) are found to be stable while low frequencies (large wavelengths) are found to be unstable, as illustrated in Figure 5 a. In the latter case, the growth of the instability is exponential, as shown in Figure 5 b.

For $w_{\text {hall }}=6 \mathrm{~mm}$ and $q_{\mathrm{m}}=5.6 \mu \mathrm{mol} / \mathrm{min}$, the threshold frequency is $f_{0}=4.25 \pm 0.05 \mathrm{~Hz}$, which corresponds to $\lambda_{0}=15 \pm 3 \mathrm{~mm}$, as shown in Figure 5 . For the unstable frequencies, the wave number appears to be proportional to the frequency, as shown in Figure 5r, indicating a celerity (pattern velocity) independent of wavelength, which means that the system is not dispersive. The measured celerity is $59 \pm 5 \mathrm{~mm} / \mathrm{s}$. The threshold frequency is measured to depend on the jet width. For $w_{\text {hall }}=12 \mathrm{~mm}$ and $q_{\mathrm{m}}=$ $12.2 \mu \mathrm{mol} / \mathrm{min}$, the threshold frequency is $f_{0}=2 \pm 0.1 \mathrm{~Hz}$ which corresponds to wavelengths of $\lambda_{0}=21 \pm 3 \mathrm{~mm}$.

\section{Rationalization of the observations}

\section{Length of the flow}

The absence of diverging flow in the unconfined area indicates that there is no surface-pressure gradient anymore in region $c$ of Figure 2. This means that the Marangoni propulsion of the surface only occurs in the region $b$ of Figure2, and stops where the flow decelerates. We deduce from this observation that the region downstream ( $c$ in Figure 2 ) is moved by the inertia accumulated in the Marangoni area ( $b$ in Figure 2).

The length of this jet can be theoretically calculated. Velocity within the Marangoni flow can be estimated from [18. to be of the order of $100 \mathrm{~mm} / \mathrm{s}\left(U_{0}=74 \mathrm{~mm} / \mathrm{s}\right.$ with the parameters used in the experiment shown in Figure 2 . ). The boundary layer develops during the Marangoni flow upstream the jet. The Marangoni flow typically extends on $L_{\mathrm{Mar}}=50 \mathrm{~mm}$, leading to a boundary layer of depth :

$$
\delta_{0}=\sqrt{\frac{\nu L_{\mathrm{Mar}}}{U_{0}}} \simeq 0.82 \mathrm{~mm}
$$

where $\nu=10^{-6} \mathrm{~m}^{2} / \mathrm{s}$ is the kinetic viscosity. This yields a Reynolds number $\mathcal{R} e=\frac{U_{0} \delta_{0}}{\nu} \simeq 50$, which indicates that the flow is inertial.

The absence of Marangoni effect in the region $c$ means that the interface is stress-free. In the jet, considering the whole boundary layer as the system, its mass increases as it thickens as the square root of time, but the vanishing velocity at the lower frontier of the boundary layer ensures no viscous force on the system. Moreover, the pressure being kept constant by the atmosphere, the pressure forces on the boundary layer simply compensate the weight : this results in a constant momentum and hence a constant product $v \delta$, where $v$ is the mean velocity of the boundary layer. 
(a)

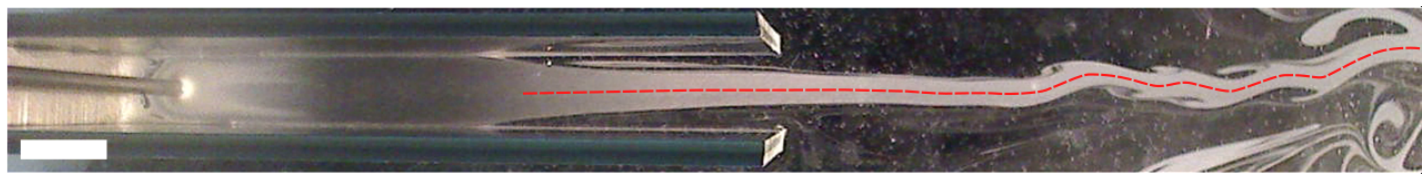

(b)

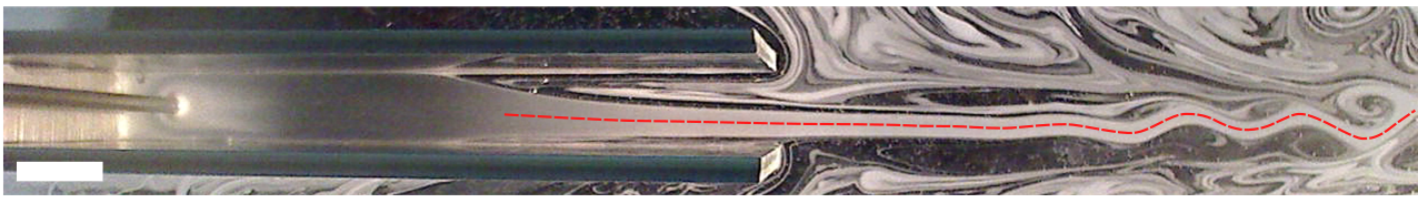

(c)

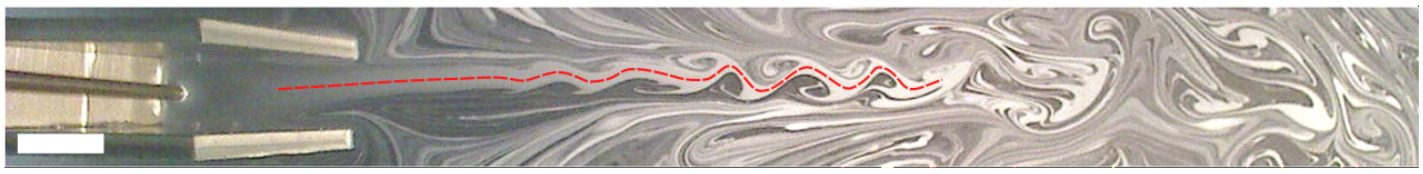

FigURE 4 - Different jets presenting various shapes during destabilization (not excited), always with a clear wavelength. The flow rate of surfactant deposition and the length of the hallway have been changed for the different pictures. Red dashed lines represent the middle line of the stream. (a) $: q_{\mathrm{m}}=14.2 \mu \mathrm{mol} / \mathrm{min}$ and $L=100 \mathrm{~mm}$. (b) $: q_{\mathrm{m}}=12.2 \mu \mathrm{mol} / \mathrm{min}$ and $L=100 \mathrm{~mm}$. (c) $: q_{\mathrm{m}}=8.1 \mu \mathrm{mol} / \mathrm{min}$ and $L=30 \mathrm{~mm}$. Scale bar $: 10 \mathrm{~mm}$.

(a)

(b)

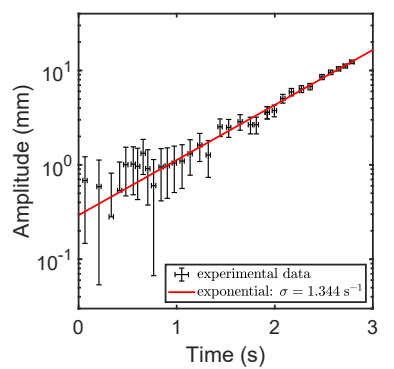

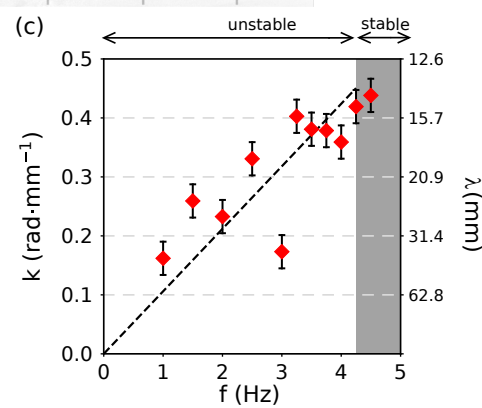

FIGURE 5 - (a) The stroboscopic observation highlights the regularity of the instability at the forced frequency. Width of hallway : $12 \mathrm{~mm}$, excitation and acquisition : $f=4 \mathrm{~Hz}$. Vertical dashed lines are guides for the eyes. Scale bar : $10 \mathrm{~mm}$. (b) Exponential growth of the amplitude for a jet of width $w_{\text {hall }}=6 \mathrm{~mm}$ excited at a frequency of $4 \mathrm{~Hz}$. The dotted line show the exponential growth with the growth rate of $1.344 \mathrm{~s}^{-1}$. (c) Wave number $k=2 \pi / \lambda$ (corresponding wavelength on the right axis) measured for the different excitation frequencies, width $w_{\text {hall }}=6 \mathrm{~mm}$ and flow rate $q_{\mathrm{m}}=5.6 \mu \mathrm{mol} / \mathrm{min}$. Dashed line represent the celerity of $59 \pm 5 \mathrm{~mm} / \mathrm{s}$.

The shape of the velocity profile is investigated through a scaling-law analysis : considering initial conditions of velocity $v_{0}$ and boundary layer $\delta_{0}$ in $x=x_{0}$, the momentum scales as

$$
p \sim v(x) \delta(x)
$$

and its conservation yields :

$$
v(x) \delta(x) \sim v_{0} \delta_{0}
$$

The vorticity oriented along $y$ scales as :

$$
\Omega \sim \frac{v}{\delta} \sim \frac{p}{\delta^{2}}
$$

The boundary-layer profile results from the advectiondiffusion of vorticity :

$$
v \frac{\partial \Omega}{\partial x}=\nu \Delta \Omega
$$

which yields

$$
\frac{p}{\delta} \frac{p}{\delta^{3}} \frac{\mathrm{d} \delta}{\mathrm{d} x} \sim \nu \frac{p}{\delta^{4}}
$$

and finally :

$$
\frac{\mathrm{d} \delta}{\mathrm{d} x} \sim \frac{\nu}{p} \sim \frac{\nu}{v_{0} \delta_{0}}
$$

It follows that the profile of boundary-layer depth and surface speed are :

$$
\delta(x)=\delta_{0}\left(1+\frac{\alpha \nu}{v_{0} \delta_{0}^{2}}\left(x-x_{0}\right)\right),
$$

and

$$
v(x)=v_{0}\left(1+\frac{\alpha \nu}{v_{0} \delta_{0}^{2}}\left(x-x_{0}\right)\right)^{-1},
$$

where $\alpha$ is a numerical prefactor that is assumed to be close to unity, and will be determined experimentally.

Equation (8) shows that even though the boundary layer grows with a square-root scaling in time, it follows a linear growth with respect to the position. Also, Equation 9 for the initial conditions $v_{0}=74 \mathrm{~mm} / \mathrm{s}$ and $\delta_{0}=0.82 \mathrm{~mm}$ in $x_{0}=0$ shows that velocity is divided by two on a length $v_{0} \delta_{0}^{2} / \nu=50 \mathrm{~mm}$ (for $\alpha=1$ ), giving a typical length scale for the jet in agreement with experiments, as shown in Figure 3. More precisely, using Equation (9), the best fit of the experimental data is shown in Figure $3 \mathrm{~d}$ by the red line and show a very good agreement between our theoretical prediction, and the measurements. The obtained values are $v_{0}=189 \mathrm{~mm} / \mathrm{s}$, and $\alpha^{-1 / 2} \delta_{0}=0.433 \mathrm{~mm}$ 
in $x_{0}=35 \mathrm{~mm}$. The theoretical depth of the boundary layer built during the Marangoni flow upstream the jet is $\delta_{0}=\sqrt{\nu x_{0} / v_{0}}=0.430 \mathrm{~mm}$, which yields the numerical prefactor $\alpha=0.986$, that can reasonably be considered equal to 1 .

The good agreement between the experimental velocity profile and the theory shows that the jet is indeed a surface flow due to the inertia accumulated in the boundary layer during the fast Marangoni flow upstream.

\section{Jet-width determination}

Here we discuss the apparent contradiction between the convergence of trajectories in Figure $3 \mathrm{a}$ and the widening of the flow in Figure 3p,c. This is common in fluid flows 33 , and comes from the choice of Lagrangian or Eulerian specification : from a Lagrangian point of view, the jet converges, as shown by the streamlines in Figure $3 a$ : a physical volume of fluid tends to go towards the center of the jet. However, from an Eulerian point of view, each moving volume transfers momentum to its neighbors, making the region of high velocity extend along the path of the jet, as visible in Figure 3 b.

This experiment shows that the jet is actually larger than what is observed with the oil-droplet tracers. Indeed, oildroplets tracers mark only the streamlines coming from the source (defining an apparent width $w_{\text {emulsion }}$ ), while particle tracking shows the whole extent of the high-speed area, defining the actual width of the jet : $w_{\text {tracking }}$.

\section{$3 \quad$ Meandering instability}

Several types of instabilities have been studied in the literature that could correspond to what is observed in these experiments : the Kelvin-Helmholtz instability [34, the viscous thread meandering 35, or folding 36, the swirling of coupled threads [37, the zig-zag instability of vertices [38, Von-Karman streets 39, or Rayleigh's development of the Kelvin-Helmholtz instability for vorticity bands [40.

Each black/white frontier in Figure 4 shows the characteristic features of a Kelvin Helmholtz instability [34, 41. The anti-symmetry of the patterns facing each other suggests that the two instabilities are coupled. These two observations and the measurements detailed previously indicate that the present phenomenon is one of the instabilities described by Rayleigh [0, and more precisely the KelvinHelmholtz destabilization of two coupled and adjacent vorticity bands, as sketched in Figure 6 .

Let's summarize the hypothesis and conclusions of Rayleigh. Considering two adjacent bands of vorticity of width $b>0$ of opposite signs, corresponding to a triangular velocity profile illustrated in Figure 6, the destabilization of the two adjacent vorticity bands is investigated. Novelty of Rayleigh's study is the non-zero width of the vorticity band (a linear velocity profile instead of a step profile), and the possible coupling between two bands. This model, initially developed to study a 3D jet, actually describes very precisely the present experiments : it corresponds to both our

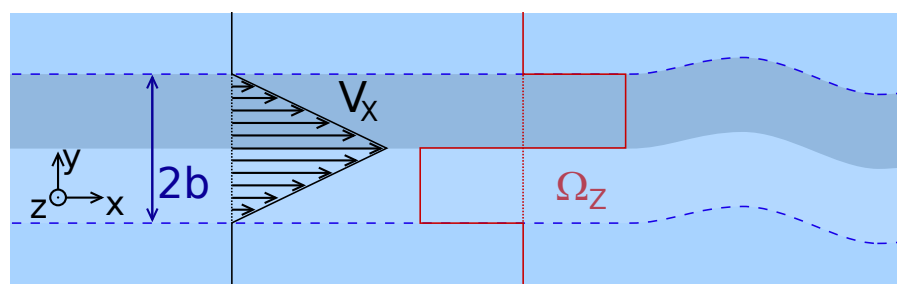

FIGURE 6 - Velocity profile (black) and vorticity profile (red) of the jet studied theoretically by Rayleigh 40 .

initial configuration (two vorticity bands) and its destabilization (meandering with long wavelengths).

The result of Rayleigh's calculations is that the two sides of the jet are coupled, and that destabilization always occurs with anti-symmetric deformations, leading to meanders growing exponentially, while all the other deformation modes are stable. Moreover, only short wavelengths $\lambda<\lambda_{0}=\pi b$ are expected to be stable, and the fastest destabilization occurs for $\lambda_{\mathrm{M}}=8 \pi b / 5 \simeq 5 b$, but the growth rate is close to its maximum on a large range of frequencies. Finally, according to the classical Kelvin-Helmholtz analysis, the instability is expected to move with a celerity which is half of the maximum velocity of the jet. This is all consistent with our observations.

Kelvin-Helmholtz instabilities perpendicular to the interface are hindered by interfacial tension and density mismatch between air and water until a threshold speed 42 . of $1.5 \mathrm{~m} / \mathrm{s}$, which is never reached in these experiments.

For the free jet as pictured in Figure 2 and 4 , as none of the modes is forced, the observed ones are the fastest, or a superposition of all the wavelengths around $\lambda_{\mathrm{M}}$. Wavelengths observed on Figure $4 \mathrm{a}\left(\lambda_{\mathrm{M}}=12.8 \pm 3 \mathrm{~mm}\right)$, Figure $4 \mathrm{~b}\left(\lambda_{\mathrm{M}}=16.5 \pm 3 \mathrm{~mm}\right)$ and Figure 4 c $\left(\lambda_{\mathrm{M}}=\right.$ $8.1 \pm 3 \mathrm{~mm}$ ) yield values for the jet width of $5.1 \pm 0.6 \mathrm{~mm}$, $6.6 \pm 0.6 \mathrm{~mm}$ and $3.2 \pm 0.6 \mathrm{~mm}$ respectively. However, due to the intrinsic low wavelength selectivity of the instability, the wavelength are not as well defined as for the excited jet (see Figure 5a).

For the forced destabilization, the measured celerity of the instability for $w_{\text {hall }}=6 \mathrm{~mm}$, extracted from Figure $5 \mathrm{~b}$, is independent of the frequency, in agreement with the Kelvin-Helmoltz theory, and equal to $c=59 \pm 5 \mathrm{~mm} / \mathrm{s}$. This value must be compared with the velocity profile from particle tracking shown in Figure 3p and Figure 3d. Particle tracking close to the jet shows velocities of the order of $3 \mathrm{~cm} / \mathrm{s}$, and the velocity inside the jet decreases from $150 \mathrm{~mm} / \mathrm{s}$ to $50 \mathrm{~mm} / \mathrm{s}$ along the flow. The celerity of the patterns corresponds to a fraction of the jet velocity, slightly different from the Kelvin-Helmholtz prediction, which is calculated for a step-profile. The threshold frequencies $\lambda_{0}$ measured in the previous section correspond to jet widths of $w_{\text {Rayleigh }}=4.8 \pm 0.6 \mathrm{~mm}$ when $w_{\text {hall }}=6 \mathrm{~mm}$, and $w_{\text {Rayleigh }}=2 b=6.7 \pm 0.6 \mathrm{~mm}$ when $w_{\text {hall }}=12 \mathrm{~mm}$. This second value can be compared with what has been measured with particle tracking and shown in Figure $3 \mathrm{p}$ $\left(w_{\text {tracking }}=6.8 \pm 0.25 \mathrm{~mm}\right)$. Particle tracking and instabi- 
lity analysis show a very good agreement on the jet width.

\section{Summary and conclusion}

Spreading of soluble surfactants occurs over a finite distance on the surface, but the effects of the Marangoni flow are observed on a much larger length scale due to the inertia provided to the fluid in the Marangoni flow. Lateral confinement of the flow can change the extent of these inertial secondary flows. Indeed, if the flow is narrow enough, as we investigated here, a huge increase of the extent of these secondary flows is observed, and the flow forms an inertial surface jet.

Flows are also induced through all the surface that converge towards the origin of the jet. Thanks to tracers inside the jet and on the whole surface, a full picture of the flow on the surface is exhibited, by combining two types of tracers providing complementary information. The velocity profile of the jet is predicted analytically and successfully compared with the experimental measurements.

Finally, the straight jet eventually destabilizes into meanders. Qualitatively, the observed features are : antisymmetric patterns (no varicose patterns), a large wavelength compared to the jet width, and eddies in the outer sides of the meanders. This instability is characterized quantitatively by forcing specific frequencies : the domain of instability is identified, and wavelength measurements show that the celerity of the patterns is independent of the frequency. These features are the signature of a situation described by Rayleigh that was never observed for surface flows yet. Quantitative comparison between the theory and the experimental observations yields a very good agreement in terms of threshold frequencies.

We have characterized and understood the 2D flows created by this configuration of soluble-surfactant directional spreading under lateral confinement. This is a first step to understand the role of inertial effects in the Marangoni flows with and without confinement. Not only this work paves the way towards a better control of the flows involved in foam and coating processes, but also it brings a new light in the study of Marangoni propulsion for natural or artificial surface micro-swimmers.

\section{Acknowledgements}

The authors thanks Aberrazzaq Moufidi for his help in capturing the surface deformation, and Isabelle Cantat and François Charru for the helpful discussions.

\section{Références}

[1] Chang Won Park. Effects of insoluble surfactants on dip coating. Journal of Colloid And Interface Science, 146(2) :382-394, 1991.

[2] Laurie Saulnier, Frédéric Restagno, Jérôme Delacotte, Dominique Langevin, and Emmanuelle Rio. What is the mechanism of soap film entrainment? Langmuir, 27(22) :13406-13409, 2011.

[3] Jacopo Seiwert, Benjamin Dollet, and Isabelle Cantat. Theoretical study of the generation of soap films : Role of interfacial visco-elasticity. Journal of Fluid Mechanics, 739 :124-142, 2014.

[4] Emmanuelle Rio and François Boulogne. Withdrawing a solid from a bath : How much liquid is coated? Advances in Colloid and Interface Science, 247 :100-114, 2017.

[5] V G Levich and V S Krylov. Surface-tension-driven phenomena. Annual Review of Fluid Mechanics, 1(1) :293-316, 1969.

[6] PR Garrett. Defoaming : theory and industrial applications, volume 45. CRC Press, 1992.

[7] Nikolai D. Denkov. Mechanisms of foam destruction by oil-based antifoams. Langmuir, 20(22) :9463-9505, 2004.

[8] D. Langevin. Rheology of adsorbed surfactant monolayers at fluid surfaces. Annual Review of Fluid Mechanics, 46(1) :47-65, 2014.

[9] V. Miralles, B. Selva, I. Cantat, and M. C. Jullien. Foam drainage control using thermocapillary stress in a two-dimensional microchamber. Physical Review Letters, 112(23) :1-5, 2014.

[10] Véronique Pimienta, Michèle Brost, Nina Kovalchuk, Stefan Bresch, and Oliver Steinbock. Complex shapes and dynamics of dissolving drops of dichloromethane. Angewandte Chemie - International Edition, 50(45): 10728-10731, 2011.

[11] Alvaro Marin, Robert Liepelt, Massimiliano Rossi, and Christian J. Kähler. Surfactant-driven flow transitions in evaporating droplets. Soft Matter, 12(5) :1593-1600, 2016.

[12] F. Wodlei, J. Sebilleau, J. Magnaudet, and V. Pimienta. Marangoni-driven flower-like patterning of an evaporating drop spreading on a liquid substrate. $\mathrm{Na}$ ture Communications, 9(1) :820, 2018.

[13] Ziane Izri, Marjolein N. van der Linden, Sébastien Michelin, and Olivier Dauchot. Self-Propulsion of Pure Water Droplets by Spontaneous MarangoniStress-Driven Motion. Physical Review Letters, 113(24):248302, dec 2014.

[14] Yasuhiro Ikezoe, Gosuke Washino, Takashi Uemura, Susumu Kitagawa, and Hiroshi Matsui. Autonomous motors of a metal-organic framework powered by reorganization of self-assembled peptides at interfaces. $\mathrm{Na}$ ture Materials, 11(12) :1081-1085, 2012.

[15] John W.M. Bush and David L. Hu. WALKING ON WATER : Biolocomotion at the Interface. Annual Review of Fluid Mechanics, 38(1) :339-369, 2006.

[16] Eric Lauga and Anthony M.J. Davis. Viscous Marangoni propulsion. Journal of Fluid Mechanics, 705 :120 133, 2012. 
[17] D. G. Suciu, Octavian Smigelschi, and Eli Ruckenstein. Some experiments on the Marangoni effect. AIChE Journal, 13(6) :1120-1124, 1967.

[18] Sébastien Le Roux, Matthieu Roché, Isabelle Cantat, and Arnaud Saint-Jalmes. Soluble surfactant spreading : How the amphiphilicity sets the Marangoni hydrodynamics. Physical Review E, 93(1) :1-13, 2016.

[19] Shreyas Mandre. Axisymmetric spreading of surfactant from a point source. Journal of Fluid Mechanics, $832: 777-792$, dec 2017.

[20] Thomas Bickel. Spreading dynamics of reactive surfactants driven by Marangoni convection. Soft Matter, 15(18) :3644-3648, 2019.

[21] Ye Tian, xiaoying Gao, Wei Hong, Miao Du, Pengju Pan, Jing Zhi Sun, Zi Liang Wu, and Qiang Zheng. Kinetic Insights into Marangoni-Effect-Assisted Preparation of Ultrathin Hydrogel Films. Langmuir, 34 :acs.langmuir.8b02626, 2018.

[22] Amar S. Basu and Yogesh B. Gianchandani. Virtual microfluidic traps, filters, channels and pumps using Marangoni flows. Journal of Micromechanics and Microengineering, 18(11) :115031, nov 2008.

[23] H. Kellay, X. L. Wu, and W. I. Goldburg. Experiments with turbulent soap films. Physical Review Letters, 74(20) :3975-3978, 1995.

[24] Michael Rivera, Peter Vorobieff, and Robert E. Ecke. Turbulence in flowing soap films : Velocity, vorticity, and thickness fields. Physical Review Letters, 81(7) :1417-1420, 1998.

[25] C. H. Bruneau and H. Kellay. Experiments and direct numerical simulations of two-dimensional turbulence. Physical Review E - Statistical, Nonlinear, and Soft Matter Physics, 71(4) :1-5, 2005.

[26] Matthieu Roche, Zhenzhen Li, Ian M. Griffiths, Sébastien Le Roux, Isabelle Cantat, Arnaud Saint-Jalmes, and Howard A. Stone. Marangoni flow of soluble amphiphiles. Physical Review Letters, 112(20) :1-5, 2014.

[27] M. M. Bandi, V. S. Akella, D. K. Singh, R. S. Singh, and S. Mandre. Hydrodynamic Signatures of Stationary Marangoni-Driven Surfactant Transport. Physical Review Letters, 119(26) :1-5, 2017.

[28] Anastasia Shmyrova and Andrey Shmyrov. Instability of a homogeneous flow from a lumped source in the presence of special boundary conditions on a free surface. EPJ Web of Conferences, 213 :02074, jun 2019.

[29] I. K. Bratukhin and L. N. Maurin. Convection inf a fluid-filling half space. PMM, 31(3) :577-580, 1967.

[30] Iu K. Bratukhin and L. N. Maurin. Stability of thermocapillary convection in a fluid filling a halfspace. Journal of Applied Mathematics and Mechanics, 46(1) :129-131, 1982.

[31] Anne D. Dussaud and Sandra M. Troian. Dynamics of spontaneous spreading with evaporation on a deep fluid layer. Physics of Fluids, 10(1) :23-38, 1998.
[32] M. M. Bandi, T. Tallinen, and L. Mahadevan. Shockdriven jamming and periodic fracture of particulate rafts. Epl, 96(3), 2011.

[33] K. R. Reddy and R. I. Tanner. Finite element solution of viscous jet flows with surface tension. Computers and Fluids, 6(2) :83-91, 1978.

[34] P. Gondret and M. Rabaud. Shear instability of twofluid parallel flow in a Hele-Shaw cell. Physics of Fluids, 9(11) :3267-3274, 1997.

[35] Stephen W. Morris, Jonathan H.P. Dawes, Neil M. Ribe, and John R. Lister. Meandering instability of a viscous thread. Physical Review E - Statistical, Nonlinear, and Soft Matter Physics, 77(6) :1-11, 2008.

[36] Thomas Cubaud and Thomas G. Mason. Folding of viscous threads in diverging microchannels. Physical Review Letters, 96(11) :1-4, 2006.

[37] Thomas Cubaud and Thomas G. Mason. Swirling of viscous fluid threads in microchannels. Physical Review Letters, 98(26) :2-5, 2007.

[38] Paul Billant and Jean Marc Chomaz. Experimental evidence for a new instability of a vertical columnar vortex pair in a strongly stratified fluid. Journal of Fluid Mechanics, 418 :167-188, 2000.

[39] E. Detemple-Laake and H. Eckelmann. Phenomenology of Kármán vortex streets in oscillatory flow. Experiments in Fluids, 7(4) :217-227, 1989.

[40] Lord Rayleigh. On the Stability, or Instability, of certain Fluid Motions. Proceedings of the London Mathematical Society, s1-11(1) :57-72, nov 1879.

[41] S. A. Thorpe. A method of producing a shear flow in a stratified fluid. Journal of Fluid Mechanics, 32(4) :693704, 1968.

[42] E Guyon, J-P Hulin, L Petit, CD Mitescu, and DF Jankowski. Physical Hydrodynamics. Applied Mechanics Reviews, 55(5) :B96-B97, sep 2002. 